\title{
PERFIL EPIDEMIOLÓGICO DOS PACIENTES ATENDIDOS NO CENTRO AUDITIVO DE PETROLINA-PE
}

\author{
Epidemiological profile of patients treated in \\ Petrolina-PE auditory center
}

\author{
Anna Tereza Pessoa da Silva Reis ${ }^{(1)}$, Fabiane Gonçalves Ferreira da Silva ${ }^{(2)}$, \\ Rodrigo Brayner de Farias ${ }^{(3)}$
}

\begin{abstract}
RESUMO
Objetivo: caracterizar o perfil epidemiológico de pacientes atendidos no Centro Auditivo de Petrolina-PE segundo sexo, idade, cidade em que residem e tipo de perda auditiva no ano de 2009. Método: participaram desta pesquisa todos os pacientes atendidos no Centro Auditivo de Petrolina-PE no ano de 2009, totalizando 553 indivíduos. Foram considerados apenas os prontuários com dados completos e exames audiológicos realizados, sendo este o critério de inclusão no estudo. Foram excluídos 73 indivíduos que tinham os prontuários incompletos. Resultados: amostra foi composta por 480 prontuários, destes, 55,2\% (265) eram do gênero feminino e 44,8\% (215) do gênero masculino. Em relação a faixa etária, observou-se que a média de idade foi de 49,1 anos, variando entre 4 e 93 anos. A distribuição da população segundo o tipo de perda auditiva foi de $220(22,9 \%)$ orelhas com limiares auditivos normais, 572 (59,5\%) com perda auditiva sensorioneural, $134(13,9 \%)$ mistas e $34(3,5 \%)$ condutivas. Em relação a cidade de origem dos participantes observou-se que $392(81,6 \%)$ indivíduos residem em Petrolina e $82(18,4 \%)$ em outras cidades de Pernambuco. Conclusão: o presente estudo demonstrou que a deficiência auditiva predominantemente foi do tipo sensorioneural. Em relação ao gênero, houve predomínio do feminino. Apresentou-se considerável número de pessoas com deficiência auditiva acima dos 60 anos, mostrando assim a necessidade de programa de reabilitação de idosos.
\end{abstract}

DESCRITORES: Perda Auditiva; Surdez; Epidemiologia dos Serviços de Saúde

\section{INTRODUÇÃO}

A audição exerce fundamental importância no desenvolvimento global do ser humano, uma vez que é considerada um dos seus principais sentidos. A perda desse sentido caracteriza um déficit

(1) Fonoaudióloga graduada pela Universidade Federal de Pernambuco e Pós-Graduanda (especialização) em Audiologia pela UNIME - União Metropolitana de Educação e Cultura.

(2) Fonoaudióloga graduada pela Universidade Federal da Bahia e Pós-Graduanda (especialização) em Audiologia pela UNIME - União Metropolitana de Educação e Cultura.

(3) Professor da União Metropolitana de Educação e Cultura; Mestre em Fonoaudiologia: Clínica Fonoaudiológica, pela Pontifícia Universidade Católica de São Paulo, Especialização em Fonoaudiologia, com ênfase em Audiologia, pela Universidade Federal da Bahia.

Conflito de interesses: inexistente sensorial que limita as atuações do indivíduo, tendo efeito adverso no estado funcional, na qualidade de vida, na função cognitiva e no bem estar emocional, comportamental e social ${ }^{1,2}$. O ato de escutar é um pré-requisito para a aquisição e o desenvolvimento da linguagem e da fala. Um dos principais distúrbios que pode interferir neste desenvolvimento é a deficiência auditiva ${ }^{3}$. Segundo Veras o deficiente auditivo pode ter um desenvolvimento da linguagem e do aprendizado semelhante ao do ouvinte normal, desde que seja devidamente estimulado ${ }^{4}$.

O aumento da população de idosos poderia sugerir sua associação com o aumento da esperança média de vida da população brasileira, indicador social da melhoria da qualidade de vida e bem-estar social. Entretanto, ainda que se tenha elevado a longevidade humana, isso não significa que tenham melhorado as condições objetivas de vida para o idoso. Sem dúvida alguma, a privação 
sensorial proveniente do declínio da acuidade auditiva representa uma das mais terríveis causas de isolamento social para o idoso ${ }^{4}$

No Brasil, pelo censo demográfico realizado em 2000 , constatou-se que existem aproximadamente 24,5 milhões de brasileiros com algum tipo de deficiência, o que significa $14,5 \%$ da população total, destes, 5,7 milhões declararam incapacidade, com alguma ou grande dificuldade de ouvir. ${ }^{5}$

O Ministério da Saúde publicou a Portaria GM n ${ }^{\circ}$ 2.073/04, de 28 de Setembro de 2004 que instituiu a Política Nacional de Atenção à Saúde Auditiva ${ }^{6}$. Para a organização, implantação e operacionalização das unidades que compõem as Redes Estaduais de Atenção à Saúde Auditiva, a Secretaria de Atenção à Saúde publicou duas Portarias, sendo elas: Portaria SAS/MS n ${ }^{\circ}$ 587, de 07 de outubro de 2004 que normatiza a organização e implantação das referidas Redes, e Portaria SAS n ${ }^{\circ} 589$, de 08 de outubro que visa à operacionalização dos Serviços de Atenção à Saúde Auditiva ${ }^{7}$.

A Secretaria de Saúde do município de PetrolinaPE implementou um Serviço de Atenção à Saúde Auditiva em média complexidade em 2006, onde é realizada avaliação otorrinolaringológica, diagnóstico da deficiência auditiva nas crianças, jovens, adultos e idosos e o fornecimento de aparelhos de amplificação sonora individual (AASI) pelo Sistema Único de Saúde, reabilitando, de forma específica a população deste município e casos encaminhados de toda macro regional Petrolina, que engloba 28 municípios de Pernambuco.

Tendo em vista a necessidade de pesquisas que visem conhecer a demanda de portadores com deficiência auditiva na região, como também subsidiar a realização de programas de prevenção da deficiência auditiva em hospitais e maternidades, o objetivo deste trabalho é caracterizar o perfil epidemiológico de pacientes atendidos no Centro Auditivo de Petrolina segundo sexo, idade, cidade em que residem e tipo de perda auditiva.

\section{MÉTODO}

Este estudo é do tipo transversal e observacional e foi realizada por meio da análise de prontuários de indivíduos atendidos entre janeiro e agosto de 2009 no Centro auditivo de Petrolina-PE, não havendo, portanto contato com os pacientes.

A cidade se destaca por ser a terceira maior do estado de Pernambuco. Possui, atualmente, cerca de 281.851 habitantes segundo o IBGE ${ }^{3}$.

O Centro Auditivo de Petrolina realiza serviços ambulatoriais especializados para diagnóstico, avaliação e tratamento de pessoas portadoras de deficiências auditivas, com concessão de aparelho de amplificação sonora individual (AASI) e terapia fonoaudiológica. Possui uma equipe multiprofissional formada por médicos otorrinolaringologistas, fonoaudiólogos audiologistas e terapeutas e assistente social. Realiza em média 220 atendimentos otorrinolaringológicos, com 115 exames e 60 adaptações de AASI por mês.

Participaram desta pesquisa todos os pacientes atendidos no Centro Auditivo de Petrolina no ano de 2009, totalizando 553 indivíduos. Foram considerados apenas os prontuários com dados completos, ou seja, dados pessoais, avaliação otorrinolaringológica e audiometria tonal e vocal, sendo este o critério de inclusão no estudo. Foram excluídos 73 indivíduos.

As variáveis estudadas foram: faixa etária, gênero, cidade em que residem e laudo audiológico, visando traçar o perfil epidemiológico dos indivíduos atendidos no Centro Auditivo de Petrolina.

A coleta de dados aconteceu no período de 01 a 06 de setembro de 2010, após a aprovação do Comitê de Ética em Pesquisa em seres humanos da Universidade Federal do Vale do São Francisco - UNIVASF sob número 27091016, conforme a resolução do CONEP 196/96. A Coordenadora do serviço autorizou a utilização dos dados dos pacientes, por meio da assinatura de uma carta de anuência.

A partir da análise das informações contidas nos prontuários, os dados foram analisados utilizando o programa Excel, com a apresentação dos resultados em tabelas de contingência e gráficos.

\section{RESULTADOS}

A amostra foi composta por 553 prontuários, sendo, dentre estes, apenas 480 continham exames audiométricos realizados no ano de 2009. Destes, $55,2 \%$ (265) eram do gênero feminino e $44,8 \%$ (215) do gênero masculino.

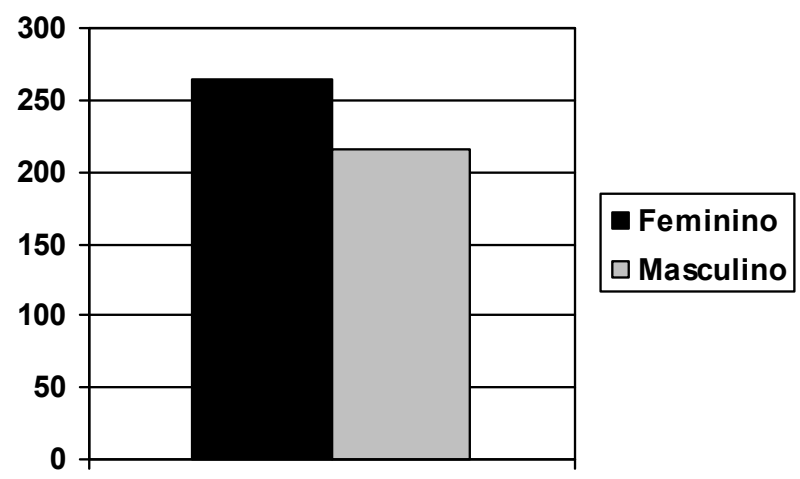

Figura 1 - Distribuição da população segundo o sexo 
Em relação a faixa etária dos indivíduos submetidos a audiometria, observou-se que a média de idade foi de 49,1 anos, variando entre 4 e 93 anos, conforme mostra a tabela 1

Tabela 1 - Distribuição da população segundo a faixa etária

\begin{tabular}{lcc}
\hline Faixa etária & $\mathbf{n}$ & $\%$ \\
\hline $4-20$ & 79 & 16,45 \\
$21-40$ & 98 & 20,41 \\
$41-60$ & 119 & 24,80 \\
Acima de 60 & 184 & 38,34 \\
\hline TOTAL & 480 & 100 \\
\hline
\end{tabular}

Com relação a cidade de origem, pôde-se observar que $392(81,6 \%)$ indivíduos residem em Petrolina e $82(18,4 \%)$ em outras cidades de Pernambuco.

Tabela 2 - Distribuição da população segundo a cidade que reside

\begin{tabular}{lcc}
\hline Cidade & $\mathbf{n}$ & $\%$ \\
\hline Petrolina & 392 & 81,6 \\
Afrânio & 12 & 2,5 \\
Araripina & 02 & 0,4 \\
Belém do S. Francisco & 05 & 1,0 \\
Cabrobó & 06 & 1,2 \\
Dormentes & 06 & 1,5 \\
Ipubi & 01 & 0,2 \\
Lagoa Grande & 07 & 1,5 \\
Ouricuri & 27 & 5,5 \\
Salgueiro & 13 & 2,8 \\
Santa Cruz & 01 & 0,2 \\
Santa Maria da Boa Vista & 05 & 1,0 \\
São José do Belmonte & 01 & 0,2 \\
Terra Nova & 01 & 0,2 \\
Tuparetama & 01 & 0,2 \\
\hline Total & $\mathbf{4 8 0}$ & $\mathbf{1 0 0}$ \\
\hline
\end{tabular}

Quanto ao resultado das audiometrias distribuímos em relação ao número de orelhas, uma vez que alguns indivíduos apresentaram perdas auditivas assimétricas, sendo encontrados: 220 $(22,9 \%)$ orelhas com limiares auditivos normais, $572(59,5 \%)$ com perda auditiva sensorioneural, $134(13,9 \%)$ mistas e 34 (3,5\%) condutivas, como mostra a tabela 3.
Tabela 3 - Distribuição da população segundo o laudo da audiometria

\begin{tabular}{lcc}
\hline Laudo da Audiometria & $\mathbf{n}$ & $\%$ \\
\hline Normal & 220 & 23,0 \\
Sensórioneural & 572 & 59,5 \\
Mista & 134 & 14,0 \\
Condutiva & 34 & 3,5 \\
\hline Total & $\mathbf{9 6 0}$ & $\mathbf{1 0 0}$ \\
\hline
\end{tabular}

\section{DISCUSSÃO}

É por intermédio da audição que o ser humano consegue organizar o seu universo, entende o mundo que o rodeia, compreende o outro, interage com o meio e adquire conhecimento ${ }^{8}$. A perda desse sentido acarreta um déficit sensorial que limita significantemente estas atuações ${ }^{1,9}$.

A deficiência auditiva é uma patologia que muitos autores descrevem como grave, uma vez que pode apresentar conseqüências importantes no desenvolvimento cognitivo, social, intelectual e da linguagem dos indivíduos quando não diagnosticado no tempo correto ${ }^{10,11}$.

Quanto ao gênero notou-se que a freqüência de indivíduos do sexo feminino foi maior (Figura 1), tal resultado corrobora com dados do IBGE, onde no Brasil há um número maior de mulheres ${ }^{3}$, bem como pelo fato dessas serem mais cuidadosas com a própria saúde procurarem freqüentemente os serviços de saúde para realização de exames, diferentemente da população masculina ${ }^{12}$.

Com relação a distribuição segundo a faixa etária, verificou-se uma prevalência maior de indivíduos acima de 60 anos. Este fato pode ser explicado pelo aumento do número de pessoas idosas - população com 60 anos ou mais ${ }^{13}$ - em todos os países do mundo, o que leva a uma maior demanda aos serviços de atenção básica à saúde ${ }^{14}$, decorrente do aumento na incidência de doenças crônicas não-transmissíveis ${ }^{15}$.

De acordo com a política nacional de saúde do portador de deficiência, ${ }^{16}$ a presbiacusia é uma perda auditiva relacionada ao envelhecimento e é apontada como principal causa de perda de audição entre adultos. Sua prevalência é estimada em torno de $30 \%$ na população idosa, definida pelas pessoas acima de 60 anos $^{17}$.

O estudo fonoaudiológico voltado para problemas auditivos na terceira idade vem crescendo uma vez que a fonoaudiologia precisa atualizar-se juntamente com as necessidades da população. As alterações auditivas na população idosa vão desde a diminuição nos limiares de audibilidade 
até dificuldades importantes na compreensão da fala, levando o indivíduo a ter problemas na comunicação e, conseqüentemente, retração e isolamento social. Em função destas limitações trazidas pela deficiência auditiva, o diagnóstico precoce faz-se primordial, a fim de diminuir o impacto na relação social com o meio ${ }^{18}$.

De acordo com a cidade em que os indivíduos residem a maior parte é da cidade de Petrolina devido a facilidade de acesso ao Centro Auditivo da cidade.

Em relação ao tipo de perda, encontrou-se maior prevalência de perda auditiva sensorioneural, corroborando com alguns autores que afirmam que a presbiacusia é definida como uma perda auditiva sensorioneural, com o grau podendo variar de leve a profundo tanto nas frequências baixas quanto nas frequências altas, apresenta início gradual e progressivo, de forma simétrica, descendente e bilateral para sons em frequências altas ( $3 \mathrm{a} 8 \mathrm{KHz}$ ), muitas vezes acompanhada por dificuldades no reconhecimento de fala ${ }^{19,20,21}$.

O Aparelho de Amplificação Sonora Individual (AASI), ou prótese auditiva, é utilizado para minimizar os efeitos negativos da deficiência auditiva nessas pessoas, sendo sua indicação a conduta médica usual e ficando sob a responsabilidade do profissional fonoaudiólogo a realização do processo de seleção, indicação e adaptação do aparelho auditivo 22 .

Em estudo realizado por Teixeira (2008) observou-se que, em pouco tempo de estimulação sonora com o AASI, $68 \%$ dos indivíduos que participaram da pesquisa, perceberam uma melhora significante na sua qualidade de vida ${ }^{23}$.

Para finalizar, é importante frisar a necessidade de implantação de programas de prevenção e diagnóstico precoce da deficiência auditiva e da sua etiologia, bem como de conscientização dos pais, da população em geral, autoridades e profissionais da saúde.

\section{CONCLUSÃO}

A partir desta pesquisa verificou-se a prevalência de $55,2 \%$ dos indivíduos que freqüentaram o Centro auditivo de Petrolina são do sexo feminino, residem no município em que o Centro Auditivo está localizado, um grande número de pessoas com deficiência auditiva acima de 60 anos, tendo como principal tipo a perda auditiva sensórioneural, mostrando assim a necessidade de programa de reabilitação de idosos.

\begin{abstract}
Purpose: to characterize the epidemiological profile of patients attending the Hearing Center of Petrolina-PE by gender, age, city where they reside and type of hearing loss, in 2009. Method: all patients treated at the Hearing Center of Petrolina-PE in 2009 took part in this study, totaling 553 subjects. We considered only the records with complete data and audiological findings, with this being the criteria for inclusion in the study. We excluded 73 subjects whose records were incomplete. Results: the sample consisted of 480 medical records, namely: $55.2 \%$ (265) were female and $44.8 \%$ (215) male. Regarding age, we noted that the mean age was 49.1 years, ranging between 4 and 93 years. The population distribution according to type of hearing loss was $220(22.9 \%)$ ears with normal hearing thresholds, 572 (59.5\%) with sensorineural hearing loss, $134(13.9 \%)$ and 34 mixed (3.5\%) conductive. Regarding the city of origin of the participants, the study showed $392(81.6 \%)$ subjects residing in Petrolina and $82(18.4 \%)$ in other Pernambuco cities. Conclusion: this study showed that hearing loss was predominantly sensorineural. Regarding gender, there was a female predominance. there was a considerable number of people with hearing loss over 60 year old, and such being the case, showing the need for a rehabilitation program for the elder.
\end{abstract}

KEYWORDS: Hearing Loss; Deafness; Health Services Epidemiology 


\section{REFERENCIAS}

1. Mulrow CD, Aguilar C, Edicott JE, Vélez R, Tuley MR, Charlip WS, Hill JA. Association between hearing impairment and the quality of life of elderly individuals. Journal of the America Geriatric Society 1990;38:45-50.

2. Butugan O, Santoro PP, Rezende E, Silveira JA, Medicis JA, Grasel SS. Diagnóstico precoce da deficiência auditiva no primeiro ano de vida de crianças com alto risco através de audiometria de tronco cerebral. Pediatria (São Paulo). 2000;22(2):115-22.

3. Gatto CL, Tochetto TM. Deficiência Auditiva Infantil: implicações e soluções. Revista CEFAC, São Paulo. 2007; 9(1): 110-5.

4. Veras RP, Mattos LC. Audiologia do envelhecimento: revisão da literatura e perspectivas atuais. Rev. Bras. Otorrinolaringol., São Paulo, 2007; 73(1) :128-34.

5. IBGE - Instituto Brasileiro de Geografia e Estatística. Síntese dos indicadores sociais 2000. Rio de Janeiro: IBGE; 2001. 369p. (Estudos e pesquisas. Informação demográfica e socioeconômica, 5).

6. Portaria MS/GM № $2073-28$ DE SETEMBRO DE 2004. Manual de Legislação de Saúde da Pessoa com Deficiência, Brasilia DF, 2 ed., 2006.

7. Portaria MS/SAS № 589- 8 OUTUBRO 2004. Manual de Legislação de Saúde da Pessoa com Deficiência, Brasilia DF, 2 ed., 2006.

8. Lichtig I, Monteiro RG, Couto MIV, Haro FMB, Campos MSC, Vaz FAC, Okay Y. Avaliação do comportamento auditivo e neuropsicomotor em lactentes de baixo peso ao nascimento. Rev. Assoc. Med. Bras. 2001; 47(1): 52-8.

9. Françozo MFC, Masson GA, Rossi TRF, Lima MCMP, Santos MFC Adesão a um Programa de Triagem Auditiva Neonatal. Saude soc., São Paulo, 2010; 19(4): 910-8.

10. Azevedo MF Avaliação audiologica no primeiro ano de vida. In: Lopes Filho O., organizador. Tratado de Fonoaudiologia. São Paulo; Roca. 2007; p. 604-16.

http://dx.doi.org/10.1590/S1516-18462011005000066

RECEBIDO EM: 22/09/2010

ACEITO EM: 02/04/2011

Endereço para correspondência:

Anna Tereza Pessoa da Silva Reis

Rua Manoel Clementino, № 1157 - Centro

Petrolina - PE

CEP: $56302-170$

E-mail: annaterezareis@yahoo.com.br
11 Baraldi GS, Almeida LC, Borges ACC. Evolução da perda auditiva no decorrer do envelhecimento. Braz J Otorhinolaryngol. 2007;73(1):64-70.

12. Pinheiro RS, Viacava F, Travassos C, Brito AS, Gênero, morbidade, acesso e utilização de serviços de saúde no Brasil. Ciênc. saúde coletiva 2002; 7(4): 687-707.

13. Organización Panamericana de la Salud. La salud en las Américas, 1998. Washington, D.C.: OPS; 1998.

14. Henry CJ, Varakamin C, Webster-Gandy J, Ulijaszek S. Anthropometry of two contrasting populations of Thai elderly living in a rural setting. Archives of Gerontology and Geriatrics 2001; 33:255-63.

15. Giatti L, Barreto SM. Saúde, trabalho e envelhecimento no Brasil. Ad Saúde Pública 2003; 19(3):759-71.

16. Mattos LC, Veras RP, A prevalência da perda auditiva em uma população de idosos da cidade do Rio de Janeiro: estudo seccional. Rev. Bras. Otorrinolaringol; 2007;75(5):654-9.

17. Carmo LC, Silveira JAM, Marone SAM, D'Ottaviano FG, Zagati LL, Lins EMDVS et al. Estudo audiológico de uma população idosa brasileira. Rev. Bras. Otorrinolaringol. . 2008; 74(3): 342-9.

18. Baraldi GS, Almeida LC, Borges ACC. Evolução da perda auditiva no decorrer do envelhecimento. Rev. Bras. Otorrinolaringol - São Paulo; 2007;73(1): 64-70.

19. Guarinello AC; Cruz MCM; O perfil dos idosos protetizados na Clínica de Fonoaudiologia da Universidade Tuiuti do Paraná. Revista Fono atual; São Paulo, 2006:8(35): 59-64.

20 Barros PF, Queiroga BA. As dificuldades encontradas no processo de adaptação de aparelho de amplificação sonora individual em indivíduos idosos. Rev CEFAC. 2006;8(3):375-85.

21 Almeida EOC, Costa CB, Oliveira SRT. Umeoka MTH. Audiometria Tonal e Emissões OtoacústicasProdutos de Distorção em Pacientes Tratados com Cisplatina. Arq Int Otorrinolaringol. 2006;10(3):1-7. 22. Korn GP, Wecky LLM. Distúrbios da audição no idoso. RBM Rev Bras Med. 2006;63(7):353-6

23. Teixeira CF, Augusto LGS, Neto SSC. Prótese auditiva: satisfação do usuário com sua prótese e com seu meio ambiente. Revista CEFAC. 2008;10(2):245-53. 